\title{
SIMULATION OF THE ELASTIC BEHAVIOUR OF THE HUMAN MANDIBLE: EXPERIMENTAL VALIDATION AND MATERIAL PARAMETER IDENTIFICATION
}

\author{
Christian Clason ${ }^{1,2}$ And Andreas M. Hinz ${ }^{1,3}$
}

\begin{abstract}
A numerical simulation of the elastic behaviour of the human mandible is realized via the finite element method. To test the validity of the simplifying assumptions of this model, carefully designed in vitro experiments are implemented on a special-purpose biomechanical test stand. Using this validation procedure, a method for identifying the material properties of the mandible can be implemented by solving an inverse problem.
\end{abstract}

Résumé. Une simulation numérique du comportement de la mandibule humaine supposée élastique a été réalisée à l'aide de la méthode des éléments finis. Pour tester la validité des hypothèses simplificatrices du modèle, des expériences soigneusement préparées ont été menées sur un banc d'essai biomécanique. En se basant sur cette validation, une méthode permettant d'identifier les propriétés matériels de la mandibule peut être implementé en résolvant un problème inverse.

\section{INTRODUCTION}

During the reconstructive surgery of the mandible, osteosynthesis plates are used to set fractured bone. The individual differences in topology and morphology makes fitting these plates to the bone, which is often badly accessible, difficult. Also, the long term stability of the implant is essential for a swift recovery. Therefore, computer-assisted planning of the surgery based on a finite element analysis could help the surgeon with the optimal insertion of these plates.

However, to be useful, the results of such a simulation must be very close to the in-vivo situation; hence experimental validation is necessary to be able to use these results with confidence. For that reason, results of numerical simulations of the elastic behaviour of the human mandible are compared to measurements from closely matched biomechanical experiments on a specially-made test unit, using cadaverous specimen.

For the simulation to give accurate results, the individual circumstances have to be carefully replicated. In our case, the complicated geometry of the mandible has to be taken into account, as well as its material properties.

Since the determination of the latter is fraught with difficulties, and the literature full of conflicting statements, we propose a method for their identification based on the experimental procedure. This method has the advantage of being non-destructive, as well as reflecting parameters relevant for functional loading. We arrive at an inverse problem, which has to be solved iteratively.

\footnotetext{
1 Zentrum Mathematik, Technische Universität München

2 e-mail: clason@appl-math.tu-muenchen.de

3 e-mail: hinz@appl-math.tu-muenchen.de
} 


\section{Materials and Methods}

Our simulation is based on the model of linear elasticity. For the beginning, the material is assumed to be homogeneous and isotropic. This system of partial differential equations is then solved by the method of finite elements, using a software package developed at our institute. To achieve an optimal representation of the individual geometry of the mandible, the finite element grid as well as the boundary conditions were taken from a CAT scan of a human specimen. The volumetric data were first segmented with the visualization software $\operatorname{amira}^{\mathrm{TM}}$ (Konrad-Zuse-Zentrum für Informationstechnik Berlin, [1]), identifying the bone and the muscular attachments. From this data, a tetraeder mesh was created by marching cubes and advancing fronts methods, and the boundary conditions defined. Muscles were taken as Neumann boundary conditions, with physiological forces taken from the literature [4]. The condyles were supposed to be fixed, giving homogeneous Dirichlet conditions there. Using these data, displacements were calculated for the loaded mandible.

The experimental validation was conducted on a purpose-made test stand constructed at the Department of Biomechanics of the Clinic for Orthopaedics and Sport Orthopaedics of the Technical University Munich [5]. Computer controlled hydraulic pistons can create arbitrary dynamic loading situations, providing the needed flexibility to match the simulated conditions. Therefore, the previously scanned mandibles used for the numerical simulation were subjected to the loads defined there. The resulting deformations are recorded by tracking markers with several digital cameras. A computer software then calculates the three-dimensional displacements, providing contact-free high-resolution measurements.

Calculating the material parameters, in our case the Lamé constants $E$ and $\nu$, from the measured displacement leads to an inverse problem, which can be formulated as follows:

Given the norm of the measured displacements of the tracked markers $u^{\delta}$ (the $\delta$ denoting the fact that these measurements are subject to noise), find the set of material parameters $x^{\delta}=(E, \nu)$ which solve the equation

$$
T\left(x^{\delta}\right)=u^{\delta}
$$

Here the operator $T$ denotes calculating the norm of the displacement of the markers via the finite element simulation, with the given material parameters $x^{\delta}$. The norm of the displacement vector was chosen to give independence from the coordinate systems used for measurement and simulation. Correct scaling was ensured.

The solution of (1) is carried out iteratively by Newton's method:

$$
T^{\prime}\left(x_{k}^{\delta}\right)\left(x_{k+1}^{\delta}-x_{k}^{\delta}\right)=u^{\delta}-T\left(x_{k}^{\delta}\right)
$$

Since this problem is ill-posed, additional regularization has to be performed for the scheme to be stable. Tikhonov regularization of (2) leads formally to a Levenberg-Marquardt iteration for the material parameters [2]:

$$
x_{k+1}^{\delta}=x_{k}^{\delta}+\left(\left(T^{\prime}\left(x_{k}^{\delta}\right)\right)^{T}\left(T^{\prime}\left(x_{k}^{\delta}\right)\right)+\alpha_{k} \mathbf{I}\right)^{-1}\left(T^{\prime}\left(x_{k}^{\delta}\right)\right)^{T}\left(u^{\delta}-T\left(x_{k}^{\delta}\right)\right) .
$$

Here, $\alpha_{k}$ denotes a regularization parameter which has to be chosen carefully to ensure convergence of the method. Starting with $\alpha_{0}=1 / 2$, the following choice was used for each iteration step:

$$
\begin{aligned}
\alpha_{k+1}=\frac{\alpha_{k}}{2}, & \text { if }\left\|u^{\delta}-T\left(x_{k+1}^{\delta}\right)\right\|<\left\|u^{\delta}-T\left(x_{k}^{\delta}\right)\right\|, \\
\alpha_{k+1}=2 \alpha_{k}, & \text { otherwise. }
\end{aligned}
$$

In practice, the iteration (3) was treated implicitly by solving the corresponding system of linear equations. The Jacobi matrix $T^{\prime}\left(x_{k}^{\delta}\right)$ was approximated by finite differences. As initial values, material parameters were taken from the literature, leading to the choice of $x_{0}=\left(1.5 \cdot 10^{6}, 0.33\right)$. This corresponds to a Young modulus of $15 \mathrm{GPa}$ and a Poisson ratio of 0.33 .

Implementation of the minimization routine was carried out in Matlab. The stopping conditions of the iteration were $\left\|T\left(x_{k+1}^{\delta}\right)-T\left(x_{k}^{\delta}\right)\right\| \leq \varepsilon$ with a given $\varepsilon=10^{-6}$, or the maximum number of iterations $k_{\max }=100$. 


\section{Results}

The method proposed was used to determine the material properties of several human cadaverous mandibles. Results were obtained which showed good agreement with previously reported values for human mandibular bone [3]. Further simulations with the calculated parameters matched the deformations which could be expected from clinical experience, giving further validation.

\section{Discussion}

This study shows that the method presented here for the identification of material parameters is viable. Besides being non-destructive, it can also easily be extended to anisotropic and inhomogeneous models, which more accurately reflect the elastic behaviour of human bone.

Furthermore, the pipeline described in Section 1 for the numerical simulation has proven very efficient in giving a correct representation of the mandibular geometry. By generating the finite element grid and the boundary conditions directly from the CAT scan's volume data, as little of the vital information as possible is lost.

Further integration of these steps into a common software framework will give surgeons a powerful tool for the real-time planning of reconstructive surgery.

\section{REFERENCES}

[1] amira. http://amira.zib.de.

[2] Heinz W. Engl, Martin Hanke, and Andreas Neubauer. Regularization of Inverse Problems. Kluwer Academic Publishers, Dordrecht, 1996.

[3] C.S. Jørgensen and T. Kundu. Measurement of material constants of trabecular bone: a micromechanical analytic study using a $1 \mathrm{Ghz}$ acoustic microscope. Journal of Orthopaedic Research, (20):151-158, 2002.

[4] Christophe Meyer, Jean-Luc Kahn, Philippe Boutemy, and Astrid Wilk. Determination of the external forces applied to the mandible during various chewing exercises. Journal of Cranio-Maxillofacial Surgery, (26):331-341, 1998.

[5] Heinrich Schieferstein, Andreas Neff, Andreas Kuhn, Hans-Florian Zeilhofer, and Hans-Henning Horch. Experimentelle strukturanalyse des menschlichen unterkiefers. In Rahman Jamal and Hans Jaschinski, editors, Virtuelle Instrumente in der Praxis. Meßtechnik. Begleitband zum Kongreß VIP 2001, Hüthig, 2000, pages 274-281. 